\title{
The Assessment of Comprehensive Vulnerability of Chemical Industrial Park Based on Entropy Method and Matter-element Extension Model
}

\author{
Yan Jingyi, a , Zhang Lijing ${ }^{1, b}$, Tao Gang ${ }^{1, \mathrm{c}}$, Jiang Shu ${ }^{2, \mathrm{~d}}$ and Guo Hanyu ${ }^{1, \mathrm{e}}$ \\ ${ }^{1}$ College of safety science and engineering, Nanjing Tech University, Nanjing \\ ${ }^{2}$ Nanjing boiler and pressure vessel inspection institute, Nanjing \\ a330720125@qq.com, ${ }^{\mathrm{b}}$ zhanglj@njtech.edu.cn, ${ }^{\mathrm{c}}$ taogangs@163.com, ${ }^{\mathrm{d}}$ shujiang@njtech.edu.cn, ${ }^{\mathrm{e}} 781994362 @$ qq.com
}

\begin{abstract}
The paper focuses on studying connotative meaning, evaluation methods and models for chemical industry park based on in-depth analysis of relevant research results in China and abroad, it summarizes and states the feature of menacing vulnerability and structural vulnerability and submits detailed influence factors such as personnel vulnerability, infrastructural vulnerability, environmental vulnerability and the vulnerability of safety managerial defeat. Using vulnerability scoping diagram establishes 21 evaluation indexes and an index system for the vulnerability evaluation of chemical industrial park. The comprehensive weights are calculated with entropy method, combining matter-element extension model to make the quantitative evaluation, then apply to evaluate some chemical industrial park successfully. This method provides a new ideas and ways for enhancing overall safety of the chemical industrial park.
\end{abstract}

Keywords: Comprehensive vulnerability evaluation; Chemical industrial park; Entropy method; Matter-element extension model

\section{Introduction}

Chemical industrial park, as the most significant embodiment of chemical concentration in recent years, brings more obvious problems. The most dangerous is high risk-taking, so it is benefit for chemical industry park to measure overall safety situation by comprehensive vulnerability evaluation based on entropy method and matter-element extension model. Through effective method, it can figure out the level of vulnerability for providing compensation measures to avoid potential risk.

The earliest studies of vulnerability can be traced back to natural disasters 0 . Now, it is becoming a hot point in evaluation of ecosystem and environment. P. Timmerman first proposed the concept of vulnerability which means the extent of a system may produce adverse effect after disasters 2. $\mathrm{Li} \mathrm{He}$ etc. systematically analyzed the concept of vulnerability 3 . Li Fengying etc. researched the vulnerability of Nanjing chemical industry based on GIS 4. Shi Peijun proposed broad and narrow disaster vulnerability assessment model 5. Li Qiujin analyzed vulnerability's concept and principle of chemical industrial park 6. Tan Zhaoyang applied fuzzy comprehensive method and PCA method to build area vulnerability by SVM (Support Vector Machine) 7. Chemical industrial park as target of regional vulnerability, it is necessary to introduce the comprehensive vulnerability assessment. At present, evaluation are most based on risk and it seldom considered the recovery of facilities, personnel density and other factors, however, the assessment of comprehensive vulnerability of chemical industrial park will benefit to monitor the dynamic management of chemical industrial park. Therefore, in this paper, it uses improved analytic hierarchy process (IAHP) combined with entropy method to determine the weight of each index layer, and then applies to Shizhuang chemical industrial park to get each level's correlation and the final vulnerability level of this chemical industrial park.

\section{Matter-element Extension Model and Method}

Matter element analysis method is used to solve the study of incompatibile problems, but this is often a difficult point in study, because it must consider things, characteristics and the corresponding quantity, and make a formal process to resolve conflicts. Currently, the matter element analysis method has gradually been applied in engineering fields including chemical industrial parks $[8,9]$. But the concept of vulnerability in chemical industrial park is fuzzy, so many comprehensive vulnerability assessment design index, and often the incompatibility between single evaluation index, so this article will be based on the fuzzy matter-element extension model to build the suitable 
comprehensive vulnerability evaluation indexes for chemical industrial park.

\section{Establish the Fuzzy Matter-element Matrix}

We will take the vulnerability of chemical industrial park as the evaluation object, namely the context in the matter-element analysis, represented by $M$. The set of evaluation index is represented by $C$, its value is $u$. For $M$, which has $\mathrm{n}$ index, then the set $C$ is expressed by $C$, $C=\left(C_{1}, C_{2}, \cdots, C_{n}\right)$, assuming that the $n$-th value of evaluation index $C_{n}$ is $u_{n}$, so $u=\left(u_{1}, u_{2}, \cdots, u_{n}\right)$. The evaluation object $M$, the evaluation index set $C$, the corresponding evaluation indexes values $u$, these three components form an orderly triple $R=(M, C, u)$, which is classical matter-element matrix and used to describe the basic elements to be evaluated.

\section{Determine the Classical Domain and Segment Field Matter-element Matrix}

Chemical industrial park comprehensive vulnerability classic domain matrix can be $R_{j}=\left(M_{j}, C_{n}, u_{j n}\right)$. In the equation, the evaluation object $M$ will be divided into $j$ evaluation levels, namely $M=\left(M_{1}, M_{2}, \cdots, M_{j}\right)$. So, each corresponding evaluation index $C_{n}$ also has $j$ evaluation levels, then $u_{j n}=\left(a_{j n}, b_{j n}\right)$ is the standard interval for the evaluation index (evaluation parameters) of $M_{j}$, and satisfies $\left(a_{j 1}, b_{j 1}\right) \cap\left(a_{j n}, b_{j n}\right)=\varnothing, \quad(i \neq \mathrm{j})$, said any two standard intervals are incompatible. Classical field matter element matrices can be expressed as:

$$
R_{j}=\left[\begin{array}{ccc} 
& C_{1} & u_{j 1} \\
& C_{2} & u_{j 2} \\
M_{j} & C_{3} & u_{j 3} \\
& \vdots & \vdots \\
& C_{n} & u_{j n}
\end{array}\right]=\left[\begin{array}{ccc}
C_{1} & \left(a_{j 1} b_{j 1}\right) \\
C_{2} & \left(a_{j 2} b_{j 2}\right) \\
M_{j} & C_{3} & \left(a_{j 3} b_{j 3}\right) \\
& \vdots & \vdots \\
& C_{n} & \left(a_{j n} b_{j n}\right)
\end{array}\right]
$$

$M_{j}$ indicates the scope of all the evaluation level. In order to give a section domain matter-element matrix, we need to do the extension analysis on the standard interval of each evaluation level for the index $C_{k}$, to obtain the some development interval $u_{p k}=\left[a_{p k}, b_{p k}\right]$, become the segment field $k$. The expanding range $\left[a_{p k}, b_{p k}\right]$ should at least contain all the standard intervals of evaluation index $C_{k}$, means it meets the condition $\left[a_{p k}, b_{p k}\right] \supseteq$ $\cup_{1 \leq i \leq j}\left[a_{j n}, b_{j n}\right]$. To give $u_{0}$ a specific value, then call $u_{0}$ the specific value of $C_{n}$ for $R_{0}$, that the actual value of the evaluation index. The matter-element matrix of the vulnerability in chemical industrial park is expressed $\operatorname{as} R_{0}=\left(M_{0}, C_{n}, a_{0 n} b_{0 n}\right)$.

\section{Determine Correlation Function and Correlation Degree}

In order to characterize 'relevant degree' between an element and a specific element interval, introduces the concept of 'function distance' in functional analysis to quantify the 'distance' from point to interval. Thus, the distance from the point $x_{i}$ (value of the evaluation $\operatorname{index} C_{n}$ ) to the corresponding feature vector interval $x_{0 j i}=\left[a_{0 j i}, b_{0 j i}\right]$ is represented by $\rho\left(x_{i}, x_{0 j i}\right)$.

$$
\rho\left(x_{i}, x_{\rho i}\right)=\left|x_{i}-\frac{\left(a_{0 j i}+b_{0 j i}\right)}{2}\right|-\frac{\left(b_{0 j i}-a_{0 j i}\right)}{2}
$$

With $\quad\left|x_{0 j i}\right|=\left|b_{0 j i}-a_{0 j i}\right|(i=1,2, \cdots, n ; j=$ $1,2, \cdots, m) ; x_{i}, x_{0 j i}, x_{\rho i}$ respectively with the quantity of chemical industrial park vulnerability, the scope of the classical field matter-element and the range of section domain matter-element's value.

We will expand the interval $\left[x_{i}, x_{o j i}\right]$ to $\left[y_{i}, y_{o j i}\right]$, and make it meet $\left[x_{i}, x_{o j i}\right] \subset\left[y_{i}, y_{o j i}\right]$, the interval expanded $\left[y_{i}, y_{o j i}\right]$ is called $Y$, for further membership characterization between $x_{i}$ and $\left[x_{i}, x_{o j i}\right]$ interval, combined with the distance function, we can calculate the correlation $K_{i}\left(x_{i}\right)$ between $x_{i}$ and $\left[x_{i}, x_{o j i}\right]$, and determine the associated degree according to its value, shown as Eq.3.

$$
k_{i}\left(x_{i}\right)=\left\{\begin{array}{c}
-\frac{\rho\left(x_{i}-x_{0 j i}\right)}{\left|x_{0 j i}\right|}, x_{i} \in X_{0 j i} \\
\frac{\rho\left(x_{i}, x_{0 j i}\right)}{\rho\left(x_{i}, X_{\rho i}\right)-\rho\left(x_{i}, x_{0 j i}\right)}, x_{i} \notin X_{0 j i}
\end{array} \text { and } X \in Y\right.
$$

\section{The Correlation Function of Each}

\section{Level for Evaluation}

If $K_{j}^{i}\left(P_{0}\right)$ indicates the correlation between the actual value of the $\mathrm{j}$-th evaluation index $C_{n}$ and the i-th measurement standard interval for the evaluation object, $a_{i}$ indicates the weight of each evaluation index, then meet the equation:

$$
K_{j}\left(P_{0}\right)=\sum_{i=1}^{n} a_{i} K_{j}\left(x_{j}\right)
$$

If $K_{j}\left(x_{j}\right)=\max K_{j}\left(x_{j}\right), j=1,2, \cdots, m$, then the chemical industrial park vulnerability belong to level $j$. When $K_{j}\left(P_{0}\right)>0$, said chemical industrial park comprehensive vulnerability $P_{0}$ is in the some standard level range, conforms to the characteristics of this level, the size of its value is proportionate to the conformity degree; when $-1 \leq K_{j}\left(P_{0}\right) \leq 0$, said chemical industrial park comprehensive vulnerability $P_{0}$ is beyond the stone village standard level range, deviate the characteristics of this level, but have the conversion conditions, under certain conditions can conform to the standard level, and the greater its value is, the more easy to convert; when $K_{j}\left(P_{0}\right)<-1$, said chemical industrial park comprehensive vulnerability $P_{0}$ is beyond the stone village standard level range, and can't convert to the standard level 10 .

\section{Determine Relevant Weight}

This paper adopts Improved Analytic Hierarchy Process (IAHP) to quantify the incidence for each index layer and strengthen the reliability of results by entropy method to modify weight. Entropy is an ideal scale in 
different decision-making process. The less uncertainty, the lower entropy 11.

For positive indexes:

$$
P_{i, j}^{\prime}=\frac{P_{i, j}-\min P_{i, j}}{\max P_{i, j}-\min P_{i, j}}
$$

For negative indexes:

$$
P_{i, j}^{\prime}=\frac{\max P_{i, j}-P_{i, j}}{\max P_{i, j}-\min P_{i, j}}
$$

Calculate the entropy of $j$-th, the equation is expressed as:

$$
e_{j}=-k \sum_{i=1}^{n} \frac{p_{i j}}{a_{j}} \ln \left(\frac{p_{i j}}{a_{j}}\right)
$$

With $a_{j}=\sum_{i=1}^{n} p_{i j} ; k>0, k=\frac{1}{\ln (n)}, e_{j} \geq 0 ; j=1,2 \cdots, n$

Calculate the difference coefficient of $j$-th, the equation is expressed as:

$$
g_{j}=\frac{1-e_{1}}{n-E_{e}}
$$

With $E_{e}=\sum_{i=1}^{n} e_{i}, 0 \leq g_{i} \leq 1$

Using the weight to correct $W_{j}$ to get the final weight, the equation is expressed as:

$$
\theta_{j}=\frac{W_{j} w_{j}}{\sum_{j=1}^{n} W_{j} w_{j}}
$$

\section{Build the Comprehensive Vulnerability Assessment System of Chemical Industrial Park}

\subsection{Set Vulnerability Evaluation Indexes and Level of Chemical Industrial Park}

There are many influence factors for comprehensive vulnerability evaluation of chemical industrial park and they are divided to three dimensions by Vulnerability Scoping diagram (VSD). It can be concluded from analysis that dominancing and causing vulnerability's factors can be summarized as two categories for chemical industrial park, structural and threaten vulnerability indexes6. The 21 index layers are established shown as Fig.1.

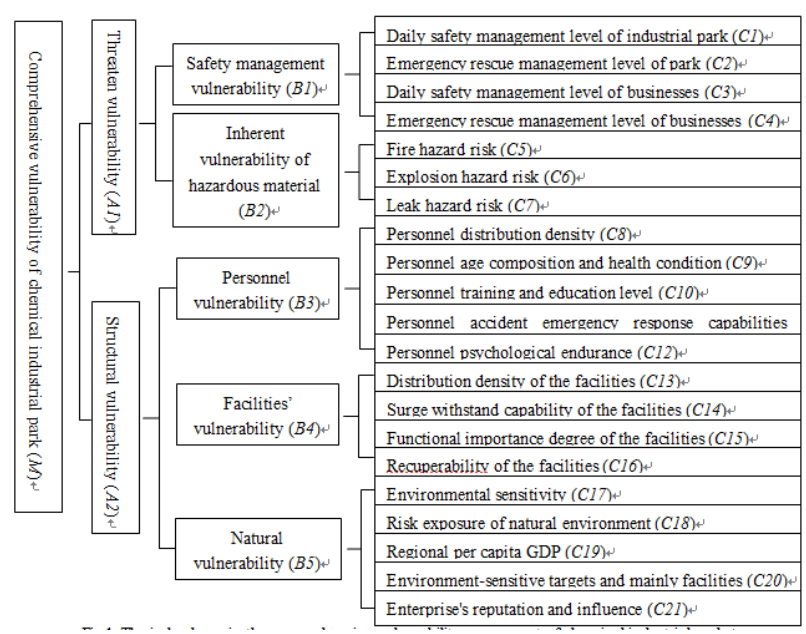

Fig.1 The index layer in the comprehensive vulnerability assessment of chemical industrial park

These 21 indexes cover all most evaluation indexes of chemical industrial park and it provides an important basis for measuring the chemical industrial park comprehensive vulnerability level accurately. This vulnerability evaluation will be divide into the following five levels: $M 1, M 2, M 3, M 4$ and $M 5$ which means very vulnerable, vulnerable, medium, safe and very safe.

\subsection{Determine Classical and Segment Field}

\section{Matter-element Matrix of the Chemical Industrial} Park

Considering the 21 indexes are difficult to be measured in a unified way, therefore, the indexes distribution was scored within the closed interval $[0,100]$ in the form of expert scoring. The higher the score gets, the safer the index is. At the same time, to divide the closed interval into five equal parts and get standard interval $\operatorname{set} C_{k}(1 \leq$ $k \leq 21) \quad: \quad\{[0,20],(20,40],(40,60],(60,80]$, $(80,100]\}$. The segment field matter-element matrix is established as bellow by matter-element extension model.

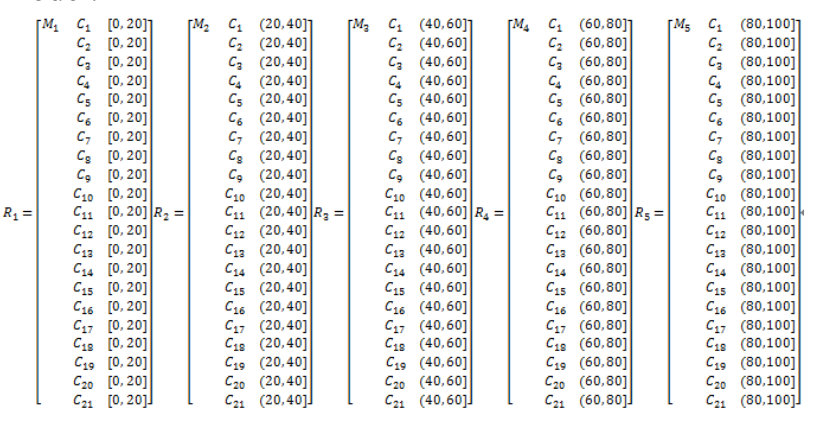

\subsection{Determine the Weights for Each Layer Index}

The weights are scored by experts for each various decision-making who marked the indexes in a range from 1 to 5 as shown in Table.1. There are $m$ experts scoring for $\mathrm{n}$ indexes that a $\mathrm{m} \times \mathrm{n}$ matrix is composed of the scores represented by $P_{i, j}$ (with $i \in 1,2, \ldots, \mathrm{m} ; j \in 1$, $2, \ldots, n)$. Standardizing the data in the matrix with Eq.5 and Eq. 6. 
Table.1 The Five-Level Scale

\begin{tabular}{c|c|c|c|c|c|}
\hline Level & 1 & 2 & 3 & 4 & 5 \\
\hline Importance & General & In between & Important & In between & Very important \\
\hline
\end{tabular}

\subsection{Case Study Applied to Shizhuang Chemical Industrial Park}

There are 25 enterprises (including storage wharfs) in Shizhuang chemical industrial park and 20 of them are chemical enterprises. There are 11 major hazard installations enterprises, 6 high-risk process enterprises, and 17 have key regulatory dangerous chemical enterprises. Shizhuang chemical industrial park has some risk, so using the matter-element extension model based on entropy weight to evaluate chemical industrial park comprehensive vulnerability is necessary.

\subsection{Determine the Weights for Each Layer Index of Chemical Industrial Park}

According to the rating value by experts for chemical industrial park, the subjective relative weight of 21 comprehensive vulnerability indexes of chemical industrial park are calculated through Eq.7 to Eq.9. The results are shown as Table.2.

Table.2 Weight Of Each Index Layer In The Comprehensive

Vulnerability Assessment

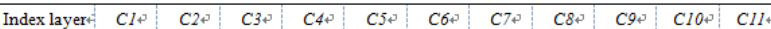

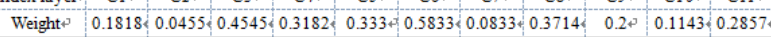

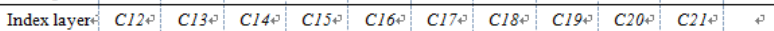

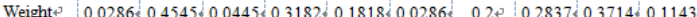

\subsection{Determine the Matter Element Matrix of Chemical Industrial Park}

Each index layer can be measured by examining meteorological, geographical, daily safety management level and emergency rescue management level of industrial park. It carries on the analysis appraisal separately from safety management vulnerability, personnel vulnerability, inherent vulnerability of hazardous material, facilities' vulnerability and environmental vulnerability, then established the matter element matrix $R_{0}$. The collection of each evaluation index actual score value is $u_{0}=[86.6,76.6,51.6,71.6,30,75,31,6.5,86.6,75,53.3,56.6$, $8.3,68.3,31.6,21.6,70,26.6]$.

\subsection{Correlation Coefficients of Different Indicators for Each Evaluation Level}

The correlation coefficients of different indicators were drawn for all indexes combined with classical field, segment field, matter-element matrix and equations. The result is shown in Table. 3 .
Table.3 The Results Of Correlation Coefficients Of Different Indicators For Each Evaluation Level

\begin{tabular}{|c|c|c|c|c|c|c|c|}
\hline$K_{j}\left(x_{i}\right)$ & very vulnerable & vulnerable & medium & safe & very safe & $\operatorname{Max} K_{j}\left(x_{i}\right)^{p}$ & Results \\
\hline$K_{1}\left(x_{1}\right)$ & -0.833 & -0.776 & -0.655 & -0.330 & 0.971 & 0.971 & very safer \\
\hline$K_{2}\left(x_{2}\right)$ & -0.708 & -0.610 & -0.415 & 0.170 & -0.127 & 0.170 & safer \\
\hline$K_{3}\left(x_{2}\right)$ & -0.395 & -0.193 & 0.210 & -0.148 & -0.369 & 0.210 & medium \\
\hline$K_{4}\left(x_{4}\right)$ & -0.645 & -0.527 & -0.290 & 0.420 & -0.228 & 0.420 & safe \\
\hline$K_{5}\left(x_{5}\right)$ & -0.250 & 0.500 & -0.250 & -0.500 & -0.625 & 0.500 & vulnerable \\
\hline$K_{6}\left(x_{6}\right)^{2}$ & $-0.687 \%$ & -0.583 & -0.375 & 0.250 & -0.166 & 0.250 & safer \\
\hline$K_{7}\left(x_{7}\right)$ & -0.268 & 0.362 & -0.210 & -0.473 & -0.605 & 0.365 & vulnerable \\
\hline$K_{8}\left(x_{8}\right)^{2}$ & 0.250 & -0.750 & -0.875 & -0.916 & -0.937 & 0.250 & very vulnerable \\
\hline$K_{9}\left(x_{9}\right)$ & -0.791 & -0.722 & -0.582 & -0.165 & 0.246 & 0.246 & very safe \\
\hline$K_{10}\left(x_{10}\right)$ & -0.666 & -0.555 & -0.332 & 0.335 & -0.200 & 0.335 & safer \\
\hline$K_{11}\left(x_{11}\right)$ & $-0.832+$ & -0.776 & -0.665 & -0.330 & 0.970 & 0.970 & very safe \\
\hline$K_{12}\left(x_{12}\right)$ & -0.687 & -0.583 & -0.375 & 0.250 & -0.166 & 0.250 & safe \\
\hline$K_{13}\left(x_{13}\right)$ & -0.418 & -0.225 & 0.162 & -0.122 & -0.363 & 0.162 & medium \\
\hline$K_{14}\left(x_{14}\right)$ & -0.457 & -0.276 & 0.085 & -0.072 & -0.350 & 0.085 & medium \\
\hline$K_{15}\left(x_{15}\right)$ & -0.791 & -0.721 & -0.582 & -0.165 & 0.246 & 0.246 & very safe \\
\hline$K_{16}\left(x_{16}\right)$ & 0.415 & -0.585 & -0.792 & -0.861 & -0.896 & 0.415 & very vulnerable \\
\hline$K_{17}\left(x_{17}\right)$ & -0.603 & -0.471 & -0.207 & 0.354 & -0.269 & 0.354 & safe \\
\hline$K_{18}\left(x_{18}\right)$ & -0.268 & 0.362 & -0.210 & -0.473 & -0.605 & 0.362 & vulnerable \\
\hline$K_{19}\left(x_{19}\right)$ & -0.068 & 0.080 & -0.460 & -0.640 & -0.730 & 0.080 & vulnerable \\
\hline$K_{20}\left(x_{20}\right)$ & -0.625 & -0.500 & -0.250 & 0.500 & -0.250 & 0.500 & safe \\
\hline$K_{21}\left(x_{21}\right)$ & -0.198 & 0.330 & -0.335 & -0.556 & -0.667 & 0.330 & vulnerable \\
\hline
\end{tabular}

According multiplication rule to calculate comprehensive correlation calculations $K_{j}\left(P_{0}\right)$ of layers and unvalued matter-element by weighted summation. The results are shown as Table.4.

Table.4 The Results Of Comprehensive Correlation Calculations Of Chemical Industrial Park

\begin{tabular}{|c|c|c|c|c|c|c|c|}
\hline Objective level & very vulnerable & vulnerable & medium & safe & very safe & Max $K_{j}\left(x_{i}\right)$ & Results \\
\hline$B 1$ & -0.577 & -0.436 & -0.155 & 0.037 & -0.078 & 0.037 & safe \\
\hline$B 2$ & -0.503 & -0.137 & -0.318 & -0.064 & -0.358 & -0.064 & safe \\
\hline$B 3$ & -0.406 & -0.723 & -0.676 & -0.415 & -0.034 & -0.034 & very safe \\
\hline$B 4$ & -0.376 & -0.451 & -0.257 & -0.274 & -0.274 & -0.257 & medium \\
\hline$B S$ & -0.350 & -0.074 & -0.309 & -0.132 & -0.501 & -0.074 & vulnerable \\
\hline$A 1$ & -0.530 & -0.247 & -0.258 & -0.027 & -0.255 & -0.027 & safe \\
\hline$A 2$ & -0.384 & -0.474 & -0.512 & -0.304 & -0.217 & -0.217 & very safe \\
\hline$M$ & -0.477 & -0.330 & -0.351 & -0.128 & -0.242 & -0.128 & safe \\
\hline
\end{tabular}

\section{Analysis of vulnerability assessment's results}

Through the calculation of comprehensive correlation degree, we can draw the conclusion that Shizhuang industrial park's comprehensive vulnerability level is safe.

The main influence factors in Shizhuang chemical industrial park are Explosion hazard risk(C6), Daily safety management level of businesses( $C 3)$, Distribution density of the facilities(C13), Personnel distribution density $(C 8)$, Environment-sensitive targets and mainly facilities $(C 20)$ according to the weight (in Table.2) and their correlation calculations' result are safe, medium, medium, very vulnerable and safe (in Table.4). It represents that personnel distribution density need to focus on. On the one hand, this park is really high concentrations that causing mass causalities is a strong possibility. Actually, Hongyuan Metal Product Co., Ltd and Kingboard Chemical Holdings Ltd are labor-intensive enterprises who need more safety supervision or move out of park when conditions permit. On the other hand, Fire hazard risk $(C 5)$ is more important than other vulnerable factors $(C 8, C 16, C 5, C 7$, C19, C21), so its key points are to strengthen the management of hazardous material because fire especially pool fire is main type of accidents in this park.

Besides that, further analysis found that although $C 5$, 
$C 7, C 18, C 19$ and $C 21$ are vulnerable, the level of these five indexes have some different. To be specific, for index $C 7$, its $M 1<M 3$ which means the level of Leak hazard risk clearly tend to be medium. And for index $C 19$, its $M 1>M 3$ which means the level of Regional per capita GDP clearly tend to be very vulnerable. In consequence, this method not only can get the vulnerability level of each index, but also can determine the tendencies according to correlation calculations for finding very vulnerable indexes in order to provide focused measures.

\section{Conclusion and Discuss}

In traditional evaluation system, the risks of explosion, fire and leakage are more focused on ,but take less attention to structural vulnerability such as personnel, facilities' and natural vulnerability. This paper puts forward quantitative methods and scoring criteria, and drawn a conclusion with Safe Level for Shizhuang chemical industrial park. According to statistics from Safety Department, there was no fatal accident in the past 5 years. The safety situation of Shizhuang industrial park is good which is identical with the result of assessment, therefore, this method is objective and practical.

Besides, this method is able to sort the indexes in a same level which is helpful for searching the vulnerable links in industrial park. As you see, establishing assessment model of comprehensive vulnerability for chemical industrial park is an important channel to make sure it run in an efficient, safe way and reduce the risk. At present, research remains to be further deepened in the recoverability of vulnerability for chemical industry park. This research establishes the foundation for further study.

\section{References}

1. Huang Jianyi, Liu Yi, Review on the Theoretical Model and Assessment Framework of Foreign Vulnerability Research. Areal Research and Development, 31(5) (2012) 1-5.

2. Peter Timmerman. Vulnerability resilience and collapse of society: A Review of Models and Possible Climatic Applications. Journal of Climatology, 1981, 1(4):396-396.

3. Li He, Zhang Pingyu, Cheng Yeqing, Concept and Assessment of Vulnerability. Progress in Geography, 27(2) (2008) 18-25.

4. Li FengYing, Bi Jun, Qu Changsheng. Whole-process environmental risk assessment and management and its application. China Environmental Science, 6 (2010) 858-864.

5. Shi Peijun. Theory on disaster science and disaster dynamics. Journal of natural disasters, 11(3) (2002) $1-9$.

6. Li Qiujin, Overview of research on Chemical industrial park vulnerability. COSHA academic conference proceedings, (2013).

7. Tan Zhaoyang. Study of Comprehensive
Assessment Methods for Chemical Industry Park Vulnerability. Nankai University, (2012).

8. Zongqin G. Construction and Development of Chemical Industrial Parks in China. International Petroleum Economics, (2004).

9. Bohle H, Downing T, Watts M. Climate change and social vulnerability: toward a sociology and geography of food in security. Global Environmental Change, 4(1) (1994) 37-48.

10. Wu Huajun, Liu Nianfeng, He Jun. Comprehensive evaluation for ecological environment based on matter-element analysis of. Journal of Huazhong University of Science and Technology, 23(1) (2006) $52-55$.

11. Zhang xianqi, Liang Chuan. Application of fuzzy matter-element model based on coefficients of entropy in comprehensive evaluation of water quality. Journal of Hydraulic Engineering, 36(9) (2005) 1057-1061.

12. Polsky C, Neff R, Yarnal B. Building comparable global change vulnerability assessments: The vulnerability scoping diagram. Global Environmental Change, 17 (2007) 472-485. 